\title{
Preliminary validity study comparing the prone lumbar hypermobility test against the prone instability test
}

\author{
Gordon LAWSON, y \\ David GRYFE \\ Ontario, Canadá.
}

\begin{abstract}
:
Objective:
\end{abstract}

The Prone Instability Test (PIT) is an established orthopaedic test that predicts the probability of low back pain patients responding positively to a spinal stabilization program (.71 sensitivity, .57 specificity for PIT). This preliminary study suggests the Prone Lumbar Hypermobility Test (PLHT) as an effective alternative to the PIT that is more suitable for a wider population due to the modified patient positioning. In contrast to the PIT, the PLHT has the patient's entire body supported by the examination table. This is hypothesized to maximize patient comfort while still maintaining clinical effectiveness for the chronic low back pain population. The purpose of this preliminary study is to determine whether the PLHT is comparable to the PIT in diagnostic effectiveness when predicting the benefits of stabilization interventions.

\section{Methods:}

To compare the clinical effectiveness of the PLHT to the PIT, each subject underwent parts I and II (relaxed and contracted) of each test (PIT and PLHT). 36 subjects received both parts of PIT and PLHT (in a randomized order).

\footnotetext{
* Este artículo obtuvo, el pasado año académico 2019-2020, el Premio María Cristina, en su séptima edición, en la modalidad de Quiropráctica. El jurado calificador estuvo conformado por: $\mathrm{D}^{\mathrm{a}}$. Ana Paula Albuquerque Facchinato Campos (Presidenta), Coordinadora de la Formación Clínica. Facultad de Quiropráctica de Los Ángeles. Southern California University of Health Sciences; D. Danilo Messa da Silva, Decano de Quiropráctica, Universidad Feevale, Novo Hamburgo, Brasil; D. Fernando Redondo Moreira Azevedo, Decano de Quiropráctica de la Universidade Anhembi-Morumbi, Sao Paulo, Brasil, y D. Ricardo Fujikawa (Secretario), Director de Estudios de Quiropráctica en el RCU Escorial María Cristina.
} 
Subjects assumed each of the four positions and $4 \mathrm{~kg} / \mathrm{cm}^{2}$ of pressure was applied directly on the skin over the L4 spinous process, using an algometer. The subjects verbally indicated perceived pain following each of the 4 positions.

Results:

Of the 36 participants included in the study, 23 participants had a negative PIT and a negative PLHT and six had a positive PIT and a positive PLHT. Three participants had a positive PIT and negative PLHT and four had a positive PLHT and negative PIT. This indicates that the PIT and PLHT have a statistically significant level of agreement.

\section{Conclusions:}

This study found that the PLHT is valid in identifying negative results in the predicted negative population, as well as positive results in the predicted positive population. For future investigations, a larger sample size is advantageous - particularly with an evenly distributed and accurate sample of positive and negative participants. This will more accurately determine the validity of the PLHT and broaden the application of the PLHT to the population for which the test is aimed to identify in clinical practice. 


\section{INTRODUCTION}

Low back pain (LBP) is one of the most prevalent conditions reported and accounts for a significant number of health care visits. Only annual physical exams, hypertension, and diabetes account for more visits than low back pain ${ }^{1}$. The 2002 National Health Interview Survey (NHIS) published data reporting the increasing prevalence of low back pain ${ }^{2}$. Back Pain ranked $6^{\text {th }}$ in overall disease burden, resulting in 83 million disability adjusted life years equal to premature mortality and years lived with disability ${ }^{3}$. The more recent publications in 2017 Lancet $^{4}$ and 2018 European Spine Journal ${ }^{5}$ rated LBP as the number one reason for disability on a global scale.

An intention of LBP assessment is for the clinician to determine the best course of treatment. One such method is the categorization of patients into subgroups, one subgroup being lumbar segmental hypermobility. Treatment results show significant improvements when using patient categorizations ${ }^{6,7}$.

The purpose of this study is to conduct a preliminary investigation into whether the novel, Prone Lumbar Hypermobility Test (PLHT) is clinically comparable to the Prone Instability Test.

\section{METHODS AND PROCEDURES}

\section{Study Design}

The Prone Instability Test (PIT) is an established orthopaedic test that predicts the probability of a low back pain patient responding positively to a spinal stabilization interventions ${ }^{6 .}$ In the PIT, the patient lies prone with the top half of their body on the examination table and their lower limbs hanging over the edge, feet resting on the ground (Figure 1). While the patient is in this relaxed position, the examiner applies posterior to anterior (PA) pressure to a lumbar spinous process. Any pain provocation during the first part of the test is reported. The second part of this test requires the patient to lift their lower limbs off the floor, through contraction of trunk musculature (Figure 2). 
While the patient is in this contracted position, the examiner applies PA pressure on the same spinous process. Again, any pain provocation during the second part of the test is reported. The PIT is considered positive if there is pain in the resting position (part I) and reduced pain in the contracted position (part II), suggesting lumbo-pelvic hypermobility.

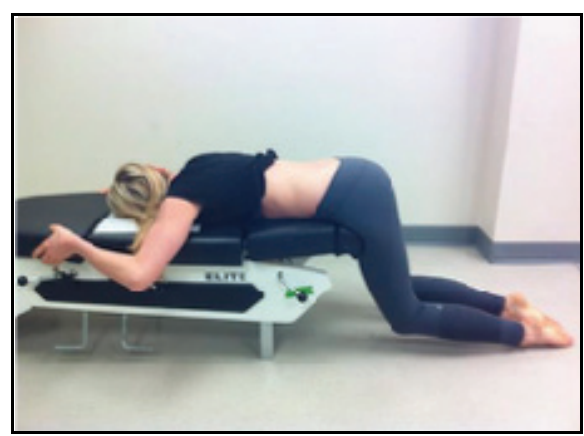

Figure 1: PIT Part I.

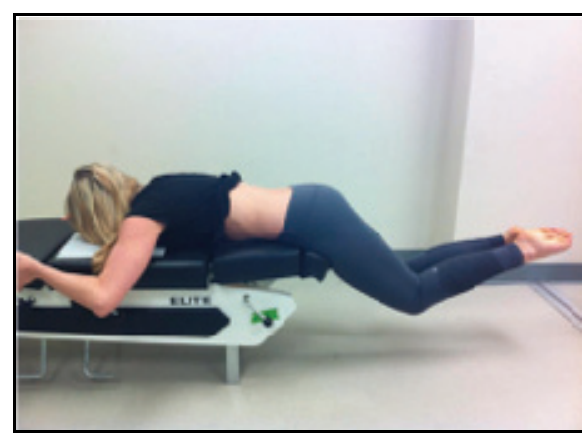

Figure 2: PIT Part II.

In this preliminary study, we suggest the PLHT as an effective alternative to the PIT. In the PLHT, the patient lies in a relaxed, prone position on an examination table with the entire body supported by the table (Figure 3). The lower limbs do not overhang the edge of the table in the PLHT. Similar to the PIT, part I of PLHT requires the examiner to apply PA pressure on a spinous process and pain provocation is recorded. Part II of the PLHT involves the contraction of trunk musculature by the patient actively lifting their lower limbs and shoulders off the examination table (Figure 4). The patient is also advised to brace by contracting the external obliques if they are familiar with this activity.

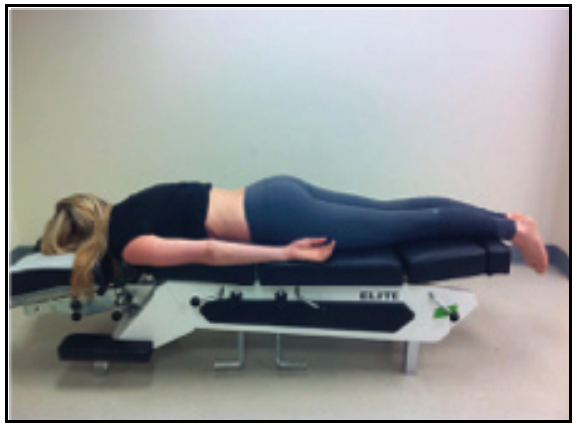

Figure 3: PLHT Part I

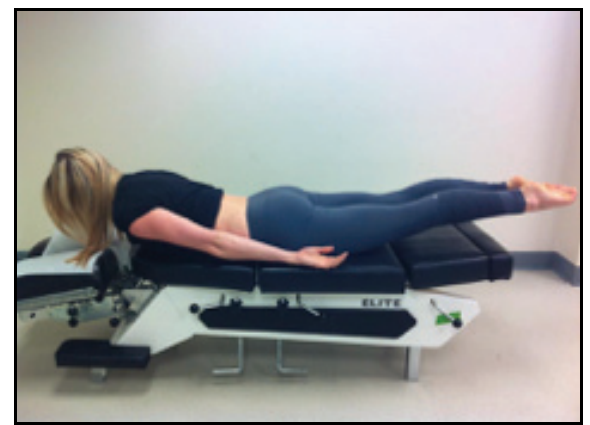

Figure 4: PLHT Part II

In order to compare the clinical effectiveness of the PLHT to the PIT, each subject underwent parts I and II (relaxed and contracted) of each test (PIT and 
PLHT). Part I of each test was administered consecutively, then part II of each test was administered consecutively. Although part I was always administered before part II, the order of which test (PIT or PLHT) was performed first was randomized for each subject. This was done to eliminate bias that may occur when comparing pain provocation between tests due to pain exacerbation from the previous test. Subjects were randomly allocated to one of four testing protocols (Table 1).

Table 1: Protocol Randomization.

\begin{tabular}{|c|l|}
\hline Group \# & Testing Protocol \\
\hline 1 & PIT part I, PLHT part I, PIT part II, PLHT part II \\
\hline 2 & PIT part I, PLHT part I, PLHT part II, PIT part II \\
\hline 3 & PLHT part I, PIT part I, PLHT part II, PIT part II \\
\hline 4 & PLHT part I, PIT part I, PIT part II, PLHT part II \\
\hline
\end{tabular}

The test administrators could not be blinded because the clinician was required to know which specific protocol to perform in order to administer the tests properly. To minimize bias, the clinician was advised not to speak with the subjects prior to, during, and after the assessment. The clinicians were not given any information regarding the study, other than how to landmark the appropriate structures (L4 spinous process) and administer the tests. In order to generate a broader sample selection, three clinicians were used instead of just one.

The subjects could not be blinded because they had to be familiar with the procedures and the testing protocol prior to administration. The student investigators instructed the subjects throughout the procedures and collected the data. The subjects rated the pain provocation as "pain" or "no pain" for part I and again for part II; if there was pain with part I, the subject was asked to rate the pain provocation of part II as "the same pain", "more pain", or "less pain" when compared to the part I position. The pain ratings were documented for all four positions and used for comparison in order to determine the level of agreement between PIT and PLHT.

\section{Sample Specification}

The study population targeted two different categories of subjects: predicted positive (predicted to have a positive PIT/PLHT) and predicted negative (predicted 
to have a negative PIT/PLHT). The study requires 40 subjects: 20 predicted positive and 20 predicted negatives. Participant categorization, as well as inclusion/exclusion criteria, was screened by the lead author. Predicted positive subjects were predicted on the basis of: i) recurrent low back pain, ii) pain that is aggravated by previous manual therapy interventions, and/or iii) pain that is mechanically aggravated by movement of the lumbar spine. Predicted negative subjects were predicted on the basis of: i) no low back pain for the last 3 months, and/or ii) a presenting complaint in the cervical or upper thoracic regions.

\section{Exclusion Criteria}

Participants were excluded if they reported any of the following: age not between 16-60 years, acute injury, cannot lie prone for minimum of 10 minutes, pain that does not change with movement or provocation, numbness and tingling or radiculopathy extending below the knee, severe constant pain that wakes them up at night, prior back surgery, infection, scoliosis, ankylosing spondylitis, pregnant, lactating, and/or post-partum females (up to a year), skin conditions (lesions, bruising, swelling) in test region, systemic diseases (such as acute cardiovascular disease, aortic aneurysms, cancer, kidney disease), using medications (such as statins, steroids, anti-coagulants, opiates, pain medications, topical creams) on the region tested.

\section{Description of Experimental Manoeuvres}

The student investigators explained the procedures of the study and demonstrated the relaxed and contracted positions to each subject before the test administrator entered the room. The subjects started by laying prone on the examination table for two minutes prior to testing. The subjects assumed each of the four positions and $4 \mathrm{~kg} / \mathrm{cm}^{2}$ of pressure (equivalent to the pressure required for finger blanching) was applied directly on the skin over the L4 spinous process, using an algometer ${ }^{8}$. Pressure was applied approximately 1 $\mathrm{kg} / \mathrm{cm}^{2} / \mathrm{second}$ and held 4 seconds in each position. Subjects verbally rated the level of discomfort by stating "pain" or "no pain". At any point during the study, the participants had the ability to stop the pressure with a verbal cue (e.g., 'stop') if it became too great. Only one of the subjects stopped the procedure due to pain, and this subject was excluded from the study.

\section{Recruitment Process and Compensation}

Subjects were recruited from existing patients at a private clinic. Subjects did not receive any payments or compensation for participation in this study. 


\section{Allocation and Minimization of Bias}

The clinicians administering the tests were trained by the lead author and given a clear guideline of what is expected from them. The clinicians were not told the purpose of the study - only that they are to administer the two tests (PLHT and PIT). Further, the examiners did not speak with the subjects before, during, or after the assessment. Results were recorded by the student investigators using a standardized form. An online randomizer was used to assign the order of each patient's test protocol. The randomized order was applied to organize the data collection forms. Each individual form was placed in a sealed envelope and was not opened until the time of testing. Only the lead author had knowledge of which patients belonged to which category (predicted positive or predicted negative) and he was not directly involved in the data collection process. No individual involved in the study simultaneously had access to all the information required to link personal information to randomization number. This information was only accessible by research personnel once data collection had been completed.

\section{Description of Outcome Measurement}

The outcome measure for this study was verbalized pain ratings from patients. The subjects rated the pain provocation as "pain" or "no pain" for part I and again for part II; if there was pain with part I, the subject was asked to rate the pain provocation of part II as "the same pain", "more pain", or "less pain" when compared to the part I position.

\section{Analysis and Justification of Sample Size}

This is a preliminary study to justify future research on this topic. According to Sackett \& Haynes (2002), in the development of a diagnostic test, phase I studies use extreme subjects on the attribute that is being measured ${ }^{10}$. Therefore, the original sample size targeted 20 predicted positive subjects and 20 predicted negative subjects, for a total of 40 subjects. According to Tractenberg et al (2010), a sample size of 25-42 is required to estimate Kappa statistics with a reasonable level of precision ${ }^{11}$. There were 37 subjects recruited for the study. Agreement between PLHT and PIT for Part I, Part II and overall was assessed by constructing two-by-two tables on whether pain was experienced for each and examining percentage agreement and percent agreement adjusted for chance using the Kappa statistic and 95\% Confidence Intervals. Kappa values were interpreted using a cut-off suggested by Streiner and Norman $(2008)^{12}$ that is anything less than 0.60 is inadequate and "even 0.75 is pushing the lower limit". 


\section{RESULTS}

Of the 37 participants recruited for the study, one participant was excluded due to significant provocation of pain (greater than 6/10) while getting into the relaxed position of the PIT. We did not continue the rest of the testing due to the level of discomfort.

Of the 36 participants included in the study, 23 participants had both a negative PIT and a negative PLHT and six had both a positive PIT and a positive PLHT. Three participants had a positive PIT and negative PLHT and four had a positive PLHT and negative PIT (Table 2). This indicates that the Prone Instability Test and Prone Lumbar Hypermobility Test have an overall percent agreement of $81 \%$ with a Kappa statistic of $\kappa=0.50$ and $95 \%$ CI $(0.18,0.82)$ (Table 3). However, it is important to recall the meaning of a positive PIT result. The overall PIT result is dependent on both part I and part II of the test. The PIT is only considered positive if there is pain in the relaxed position (part I) and then reduced pain in the contracted position (part II). Therefore, it is necessary to assess the percent agreement of each individual part. When comparing part I of each test, 18 of the 36 participants had no pain with both PIT and PLHT and 12 had pain with both PIT and PLHT (Table 2). Three participants had pain with PIT but not PLHT and three had pain with PLHT but not PIT (Table 2). This indicates an overall percent agreement of $83 \%$ for part I of the tests with $\kappa=0.66$ and $95 \% \mathrm{CI}(0.41,0.91)$. (Table 4$)$. When comparing part II of the tests, 27 of the 36 participants had no pain with both PIT and PLHT and five had a pain with both PIT and PLHT (Table 2). Two participants had pain with PLHT but not PIT and two participants had pain with PIT but not PLHT. This indicates an overall percent agreement of $89 \%$ with $\kappa=0.65$ and $95 \% \mathrm{CI}(0.33,0.96)$ for part II of the tests (Table 5).

Table 2: Collected Data

\begin{tabular}{|c|c|c|c|c|c|c|c|}
\hline Participant \# & Category & \multicolumn{2}{|c|}{ Part I } & \multicolumn{2}{c|}{ Part II } & \multicolumn{2}{c|}{ Overall Test Result } \\
\hline & & PIT & PLHT & PIT & PLHT & PIT & PLHT \\
\hline 1 & EN & no pain & no pain & no pain & no pain & - & - \\
\hline 2 & EN & pain & no pain & no pain & no pain & + & - \\
\hline 3 & EN & pain & no pain & pain & pain & - & - \\
\hline 4 & EN & no pain & no pain & no pain & no pain & - & - \\
\hline
\end{tabular}




\begin{tabular}{|c|c|c|c|c|c|c|c|}
\hline \multirow[t]{2}{*}{ Participant \# } & \multirow[t]{2}{*}{ Category } & \multicolumn{2}{|c|}{ Part I } & \multicolumn{2}{|c|}{ Part II } & \multicolumn{2}{|c|}{ Overall Test Result } \\
\hline & & PIT & PLHT & PIT & PLHT & PIT & PLHT \\
\hline 5 & EP & no pain & no pain & no pain & no pain & - & - \\
\hline 6 & $\mathrm{EN}$ & no pain & no pain & no pain & no pain & - & - \\
\hline 7 & $\mathrm{EN}$ & no pain & no pain & no pain & no pain & - & - \\
\hline 8 & $\mathrm{EN}$ & N/A & N/A & N/A & N/A & N/A & $\mathrm{N} / \mathrm{A}$ \\
\hline 9 & $\mathrm{EN}$ & no pain & no pain & no pain & no pain & - & - \\
\hline 10 & $\mathrm{EN}$ & no pain & no pain & no pain & pain & - & - \\
\hline 11 & $\mathrm{EN}$ & no pain & no pain & no pain & no pain & - & - \\
\hline 12 & $\mathrm{EN}$ & no pain & no pain & no pain & no pain & - & - \\
\hline 13 & EP & pain & pain & no pain & no pain & + & + \\
\hline 14 & EN & no pain & no pain & no pain & no pain & - & - \\
\hline 15 & $\mathrm{EN}$ & pain & no pain & no pain & no pain & + & - \\
\hline 16 & $\mathrm{EN}$ & no pain & pain & no pain & no pain & - & + \\
\hline 17 & $\mathrm{EN}$ & no pain & no pain & no pain & no pain & - & - \\
\hline 18 & EP & pain & pain & no pain & no pain & + & + \\
\hline 19 & $\mathrm{EN}$ & no pain & pain & no pain & no pain & - & + \\
\hline 20 & $\mathrm{EN}$ & no pain & no pain & no pain & no pain & - & - \\
\hline 21 & EN & no pain & no pain & no pain & no pain & - & - \\
\hline 22 & EP & pain & pain & no pain & no pain & + & + \\
\hline 23 & EP & pain & pain & no pain & pain & + & - \\
\hline 24 & EP & no pain & no pain & pain & no pain & - & - \\
\hline 25 & $\mathrm{EP}$ & pain & pain & no pain & no pain & + & + \\
\hline 26 & $\mathrm{EP}$ & pain & pain & pain & pain & - & - \\
\hline 27 & EP & no pain & no pain & no pain & no pain & - & - \\
\hline
\end{tabular}




\begin{tabular}{|c|c|c|c|c|c|c|c|}
\hline Participant \# & Category & \multicolumn{2}{|c|}{ Part I } & \multicolumn{2}{c|}{ Part II } & \multicolumn{2}{c|}{ Overall Test Result } \\
\hline & & PIT & PLHT & PIT & PLHT & PIT & PLHT \\
\hline 28 & EP & pain & pain & pain & no pain & - & + \\
\hline 29 & EP & pain & pain & no pain & no pain & + & + \\
\hline 30 & EP & no pain & pain & no pain & no pain & - & + \\
\hline 31 & EP & no pain & no pain & no pain & no pain & - & - \\
\hline 32 & EP & pain & pain & pain & pain & - & - \\
\hline 33 & EP & pain & pain & no pain & no pain & + & + \\
\hline 34 & EP & no pain & no pain & no pain & no pain & - & - \\
\hline 35 & EP & no pain & no pain & no pain & no pain & - & - \\
\hline 36 & EP & pain & pain & pain & pain & - & - \\
\hline 37 & EP & pain & pain & pain & pain & - & - \\
\hline
\end{tabular}

EN: predicted negative

EP: predicted positive

N/A: Participant excluded

Table 3: Overall Test Comparison.

\begin{tabular}{|c|c|c|}
\hline & PIT + & PIT - \\
\hline PLHT + & 6 & 4 \\
\hline PLHT - & 3 & 23 \\
\hline
\end{tabular}

Percent agreement $=(\mathrm{a}+\mathrm{d}) /(\mathrm{a}+\mathrm{b}+\mathrm{c}+\mathrm{d}) * 100$

$$
\begin{aligned}
& =(6+23) /(6+4+3+23) * 100 \\
& =81 \%
\end{aligned}
$$

Kappa $=0.50$ with $95 \%$ CI $(0.18,0.82)$ 
Table 4: Part I Comparison.

\begin{tabular}{|c|c|c|}
\hline & $\begin{array}{c}\text { PIT } \\
\text { (pain) }\end{array}$ & $\begin{array}{c}\text { PIT } \\
\text { (no pain) }\end{array}$ \\
\hline $\begin{array}{c}\text { PLHT } \\
\text { (pain) }\end{array}$ & 12 & 3 \\
\hline $\begin{array}{c}\text { PLHT } \\
\text { (no pain) }\end{array}$ & 3 & 18 \\
\hline
\end{tabular}

Percent agreement $=(\mathrm{a}+\mathrm{d}) /(\mathrm{a}+\mathrm{b}+\mathrm{c}+\mathrm{d}) * 100$

$$
\begin{aligned}
& =(12+18) /(12+3+3+18) * 100 \\
& =83 \%
\end{aligned}
$$

Kappa $=0.66$ with $95 \%$ CI $(0.41,0.91)$

Table 5: Part II Comparison.

\begin{tabular}{|c|c|c|}
\hline & $\begin{array}{c}\text { PIT } \\
\text { (pain) }\end{array}$ & $\begin{array}{c}\text { PIT } \\
\text { (no pain) }\end{array}$ \\
\hline $\begin{array}{c}\text { PLHT } \\
\text { (pain) }\end{array}$ & 5 & 2 \\
\hline $\begin{array}{c}\text { PLHT } \\
\text { (no pain) }\end{array}$ & 2 & 27 \\
\hline
\end{tabular}

Percent agreement $=(\mathrm{a}+\mathrm{d}) /(\mathrm{a}+\mathrm{b}+\mathrm{c}+\mathrm{d}) * 100$

$$
\begin{aligned}
& =(5+27) /(5+2+2+27) * 100 \\
& =89 \%
\end{aligned}
$$

Kappa $=0.65$ with $95 \%$ CI $(0.33,0.96)$

\section{DISCUSSION}

In clinical settings, the terms instability and hypermobility are sometimes used synonymously. Further, there is often a discrepancy in the use of the terms depending on the type of clinician. In the spine, orthopaedic specialists use the term instability when describing a segment that has excessive vertebral 
translation or rotation. ${ }^{13}$ Currently, radiographic measurement of sagittal translation and rotation is the gold standard for diagnosing instability. ${ }^{14}$ Hypermobility is generally determined through clinical evaluation.

With the definitions of instability and hypermobility considered, the PIT is in reality, more likely measuring hypermobility and not instability as the name suggests. A literature search revealed there are currently no studies examining modified PITs. The research does support the positive PIT is an indication that stabilization intervention is warranted ${ }^{15}$. It in truth does not identify instability nor hypermobility. Similarly, the PLHT does not assess true hypermobility but rather the indication that stabilization investigation and intervention is warranted.

The purpose of this study was to perform a preliminary evaluation of the validity of the Prone Lumbar Hypermobility Test compared to an established standard, the Prone Instability Test. The PLHT is proposed as a favourable alternative to the PIT that is more suitable for a wider population due to the modified patient positioning. Having the entire body supported on the table reduces the moment arm from which the patient must lift their lower limbs, decreasing demands on contracting muscles. This in turn will decrease the load placed on the patient's spine. The positioning for the PLHT is particularly advantageous to patients who are deconditioned and/or in pain, making the position for part II of the PIT unattainable, and therefore, reducing applicability of the test.

An algometer was used to apply pressure to the spinous process to reduce potential inconsistencies between tests. Use of the algometer allows the test administrators to standardize the amount of force applied. A study was completed using force plates to determine the validity and reliability of algometry. ${ }^{9}$ It was concluded that the tool is valid since values between the algometer and the force plate were highly correlated. The study also concluded that the use of an algometer can produce reliable results if the test administrator was experienced and had practice ${ }^{9}$. The test administrators in this study were experienced and trained in the use of the algometer.

The PLHT, which showed only modest agreement with the PIT with agreement statistic of $\kappa=0.5095 \% \mathrm{CI}(0.18,0.82)$ below the minimum acceptable cut-off of 0.60 suggested by Streiner and Norman ${ }^{12}$ is proposed as an effective alternative to the PIT particularly for deconditioned and chronic pain populations, due to the modified patient positioning. The PLHT is faster and easier to administer than the PIT, therefore, practicing clinicians may be more inclined to utilize this test. The PLHT can be seen as a predictive screening test which can be 
utilised with virtually every low back pain patient. It requires less time and effort on behalf of the patient and clinician. Tests such as the PLHT clinically categorize patients allowing clinicians to identify which patients would benefit from stabilization procedures.

The PLHT is suggested as an alternative to the PIT because it is suitable for a wider population due to the modified patient positioning. Having the entire body supported on the table reduces the moment arm from which the patient must lift their lower limbs, decreasing demands on contracting muscles. For example, the participant that was excluded from the study due to pain from part I of PIT may have been able to proceed with the PLHT, which requires less load on the spine. Unfortunately, this was not tested because the participant was relieved from the study due to increased pain from getting into the relaxed position of the PIT.

For statistical reasons described in the methodology, an adequate sample size was determined to be $25-42$ participants. The sample size used in this study should be deemed adequate since 37 participants were recruited and 36 participants were included. Of the 36 participants included, 19 were categorized as predicted positive (predicted to have a positive PIT/PLHT). The other 17 participants were categorized as predicted negative (predicted to have a negative PIT/PLHT). Of the 19 predicted positive participants, six had a positive PIT and PLHT result. This may indicate that the screening process for the predicted positive population could have been improved. For future studies, it is imperative to do a thorough screening to have a test population that equally represents both predicted positive and predicted negative populations.

The results of this study may suggest PLHT as a valid test as an alternative to the PIT; however, there are some limitations and biases in the methodology (which will be discussed later). It is suggested that the PLHT has potential to be a good initial screening test. It is hypothesized to have the ability to detect individuals who would benefit from spinal stabilization interventions. If the PLHT is positive, the clinician can move forward with the prescription of stabilization exercises. Stabilization exercises have been shown to be effective for patients with a positive $\mathrm{PIT}^{15}$. Confirmed hypermobility could also indicate a possible contraindication for manipulation ${ }^{16}$. If there are no improvements from stabilization exercises, the patient could be referred for flexion/extension radiographs to assess for instability and in severe cases, consider other interventions.

This study was approved by the Canadian Memorial Chiropractic College (Toronto, Ontario) Research Ethics Board. This study is a comparison between 
two minimally invasive orthopaedic tests, as such, the participants were exposed to minimal risk (equivalent to a physical examination). Besides the participant that was excluded, there were no side effects reported by the subjects who participated in the study.

\section{Limitations}

The randomization used detracted from the usual clinical methodology for this test. A more practical approach would have been to randomize the order of PIT and PLHT tests and perform part 1 and 2 of the full test followed by a washout period to allow subjects to fully recover from the test procedure and then performance of the other test to establish a true comparison of the two tests in their usual application.

Another limitation to this study is that three clinicians were used to perform the procedures instead of just one clinician. This was in order to generate a broader sample selection. Although the clinicians were equally trained in the procedures of the study, there may have been inter-rater inconsistencies. Future studies may consider measuring inter-rater reliability.

A further limitation to this study is the subjective nature of the outcome measure used where patients verbally rated "pain" or "no pain". Application of EMG measurements of muscle activation in follow up studies could reduce subjectivity. EMG is an established method used in spinal pain research ${ }^{11}$.

Use of the algometer also limits the results of this study. In clinical practice, the test administrator would use a thumb contact to apply the PA pressure. The contact surface of the algometer used in this study is significantly less padded compared to the hand contact. It is fair to assume that the algometer could alter perceived pain and produce different results in practice. Future studies may consider the use of force sensing table technology to standardize the amount of pressure used in testing so that the test administrator could more closely replicate the test as it would be in a clinical setting, using the clinician's thumb instead of an algometer ${ }^{17}$.

It is important to note that with a sample size of 36 , the precision on the kappa statistics is modest at best. This is evidenced by the wide confidence intervals of $\kappa$ that can range from 0 to 1 and in this case the confidence interval for main finding is quite large $(0.18,0.82)$ 


\section{CONCLUSION}

Although the Prone Instability Test is a clinically effective orthopaedic test for determining the benefit of stabilization exercises, it may not be appropriate for certain populations. Chronic low back pain, elderly, and deconditioned patients may be unable to maintain proper positioning with the PIT; therefore, the Prone Lumbar Hypermobility Test is a promising alternative screening test, which is easier to use for both the patient and clinician. This study found that the PLHT is valid in identifying negative results in the predicted negative population, as well as positive results in the predicted positive population and is therefore comparable to the PIT in identifying patients who would benefit from stabilization exercises.

The identification of patients who have positive testing would benefit from modifying therapeutic interventions. It is likely that manipulation should be avoided and stabilization the main goal. The stabilization can be provided by bracing and neutral exercises, prolotherapy, PRP and stem cell interventions, based upon the degree and hypermobility/instability and patient/clinician preference. Further research is required to identify which stabilization intervention is superior.

\section{REFERENCES}

1. Martin B, Deyo R, Mirza S, Turner J, Comstock B, Sullivan S, et al. Expenditures and health status among adults with back and neck problems. JAMA: Journal Of The American Medical Association [serial on the Internet]. (2008, Feb 13), [cited February 11, 2017]; 299(6): 656-664. Available from: CINAHL Plus with Full Text.

2. Deyo R, Mirza S, Martin B. Back pain prevalence and visit rates: estimates from U.S. national surveys, 2002. Spine (03622436) [serial on the Internet]. (2006, Nov), [cited February 20, 2017]; 31(23): 2724-2727. Available from: CINAHL Plus with Full Text.

3. Hoy D et al. The global burden of low back pain: estimates from the Global Burden of Disease 2010 Study. Ann Rheum Dis. 2014Jun;73(6):968-74.

4. Institute for Health Metrics and Evaluation (IHME). Findings from the Global Burden of Disease Study 2017. Seattle, WA: IHME, 2018.

5. Eric Hurwitz, Kristi Randhawa, Hainan Yu, Pierre Cote, Scott Haldeman The Global Spine Care Initiative: a summary of the global burden of low back 
and neck pain studies European Spine Journal (2018) 27 (Suppl 6):5796$5801 \mathrm{https}: / /$ doi.org/10.1007/s00586-017-5432-9.

6. Hall H, McIntosh G, Boyle C. Clinical Study: Effectiveness of a low back pain classification system. The Spine Journal [serial on the Internet]. (2009, Jan 1), [cited February 15, 2017]; 9648-657. Available from: ScienceDirect.

7. Fritz JM, George S The use of a classification approach to identify subgroups of patients with acute low back pain. Interrater reliability and short-term treatment outcomes. Spine (Phila Pa 1976). 2000 Jan;25(1):106-14.

8. Fischer A. Algometry in diagnosis of musculoskeletal pain and evaluation of treatment outcome: an update...presented at the symposium, 'Diagnosis and Treatment of Muscle Pain Syndromes: Myofascial, Spasm, Fibromyalgia' an official Satellite Symposium to the 8th World Congress on Pain held on August 23-25, 1996 at the University of British Columbia Conference Center in Vancouver, Canada. Journal Of Musculoskeletal Pain [serial on the Internet]. (1998, Mar), [cited March 1, 2017]; 6(1): 5-32. Available from: CINAHL Plus with Full Text.

9. Kinser A, Sands W, Stone M. Reliability and validity of a pressure algometer. Journal Of Strength \& Conditioning Research (Lippincott Williams \& Wilkins) [serial on the Internet]. (2009, Jan), [cited March 1, 2017]; 23(1): 312-314. Available from: CINAHL Plus with Full Text.

10. Sackett D, Haynes R. The architecture of diagnostic research. BMJ (Clinical Research Ed.) [serial on the Internet]. (2002, Mar 2), [cited March 1, 2017]; 324(7336): 539-541. Available from: MEDLINE with Full Text.

11. Tractenberg R, Yumoto F, Jin S, Morris J, Tractenberg R, Morris J, et al. Sample size requirements for training to a kappa agreement criterion on clinical dementia ratings. Alzheimer Disease \& Associated Disorders [serial on the Internet]. (2010, July), [cited March 1, 2017]; 24(3): 264-268. Available from: CINAHL Plus with Full Text.

12. Streiner DL, Norman GR. Health Measurement Scales: a practical guide to their development and use Fourth Edition. Oxford University Press: Toronto.

13. Magee D. Orthopedic physical assessment [monograph on the Internet]. [place unknown]: St. Louis, MO : Saunders Elsevier, 2008.; 2008. [cited February 18, 2017]. Available from: CMCC Library Catalogue (OPAC). 
14. Ferrari S, Manni T, Bonetti F, Hugo Villafañe J, Vanti C. A literature review of clinical tests for lumbar instability in low back pain: validity and applicability in clinical practice. Chiropractic \& Manual Therapies [serial on the Internet]. (2015, Apr), [cited February 5, 2017]; 23(1): 112. Available from: CINAHL Plus with Full Text.

15. Hicks GE, Fritz JM, DeLitto A, McGill SM Preliminary Development of a Clinical Prediction Rule for Determining Which Patients With Low Back Pain Will Respond to a Stabilization Exercise Program Archives of Physical Medicine and Rehabilitation Volume 86, Issue 9, September 2005, Pages 1753-1762.

16. Abbott J, McCane B, Herbison P, Moginie G, Chapple C, Hogarty T. Lumbar segmental instability: a criterion-related validity study of manual therapy assessment. BMC Musculoskeletal Disorders [serial on the Internet]. (2005, Nov 7), [cited February 9, 2017]; 656. Available from: MEDLINE with Full Text.

17. McGill S. Low back disorders: evidence-based prevention and rehabilitation [monograph on the Internet]. [place unknown]: Champaign, IL: Human Kinetics, 2016; 2007. [cited March 1, 2017]. Available from: CMCC Library Catalogue (OPAC).

18. Vear H. Chiropractic standards of practice and quality of care. Aspen Publishing Company [serial on the Internet]. (1992, Jan 1), [cited March 1, 2017]; Available from: AMED - The Allied and Complementary Medicine Database.

19. Pecos-Martín D, de Melo Aroeira A, Verás Silva R, Martínez de Tejada Pozo G, Rodríguez Solano L, Falla D, et al. Immediate effects of thoracic spinal mobilisation on erector spinae muscle activity and pain in patients with thoracic spine pain: a preliminary randomised controlled trial. Physiotherapy [serial on the Internet]. (2017, Mar 1), [cited February 19, 2017]; 1039097. Available from: ScienceDirect.

20. Triano J, Schultz A. Loads transmitted during lumbosacral spinal manipulative therapy. Spine (03622436) [serial on the Internet]. (1997, Sep), [cited February 20, 2017]; 22(17): 1955-1964. Available from: CINAHL Plus with Full Text. 
